\title{
Theoretical elastic moduli for disordered packings of interconnected spheres
}

Cite as: J. Chem. Phys. 127, 174512 (2007); https://doi.org/10.1063/1.2792995

Submitted: 10 July 2007 . Accepted: 10 September 2007 . Published Online: 07 November 2007

Alessio Zaccone, Marco Lattuada, Hua Wu, and Massimo Morbidelli

\section{ARTICLES YOU MAY BE INTERESTED IN}

Linking self-assembly, rheology, and gel transition in attractive colloids

Journal of Rheology 58, 1219 (2014); https://doi.org/10.1122/1.4878838

Rheology of viscoelastic suspensions of spheres under small and large amplitude oscillatory shear by numerical simulations

Journal of Rheology 57, 813 (2013); https://doi.org/10.1122/1.4798626

New measures for characterizing nonlinear viscoelasticity in large amplitude oscillatory shear Journal of Rheology 52, 1427 (2008); https://doi.org/10.1122/1.2970095

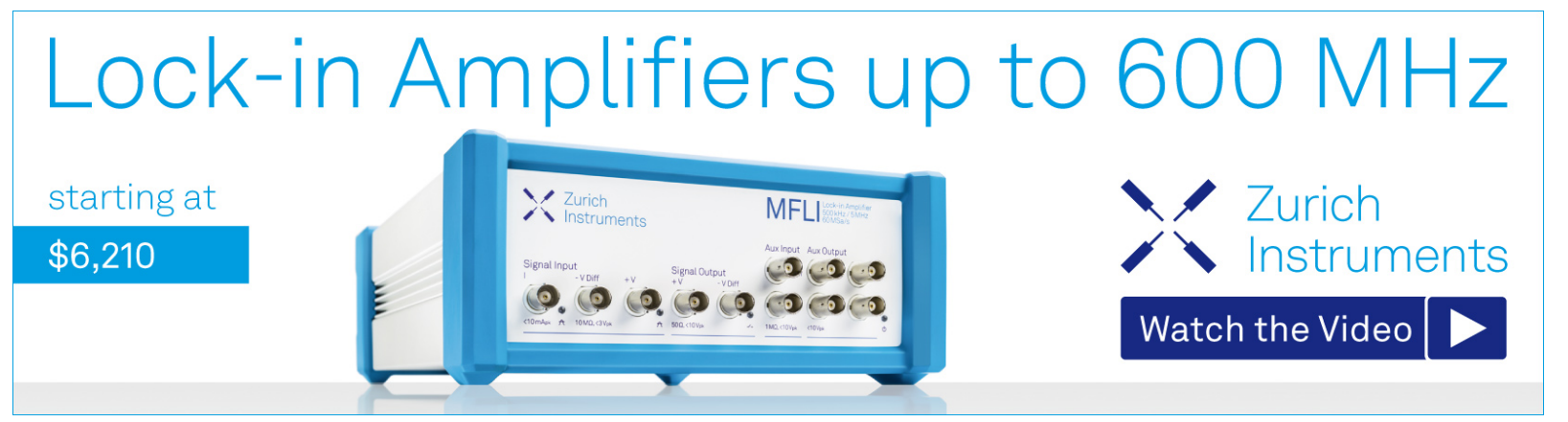

J. Chem. Phys. 127, 174512 (2007); https://doi.org/10.1063/1.2792995 


\title{
Theoretical elastic moduli for disordered packings of interconnected spheres
}

\author{
Alessio Zaccone, ${ }^{\text {a) }}$ Marco Lattuada, Hua Wu, and Massimo Morbidelli \\ Institute for Chemical and Bioengineering, Department of Chemistry and Applied Biosciences, ETH Zurich, \\ 8093 Zurich, Switzerland
}

(Received 10 July 2007; accepted 10 September 2007; published online 7 November 2007)

\begin{abstract}
A theoretical model has been developed which provides analytical expressions for the elastic moduli of disordered isotropic ensembles of spheres interconnected by physical bonds. Young's and shear moduli have been derived assuming an ideal random isotropic network and the radial distribution function for disordered packings of spheres. The interparticle interactions are accounted for in terms of surface forces for the two distinct cases of perfectly rigid spheres and spheres deformable at contact. A theoretical expression is also derived in a similar way for the bulk or compressibility modulus. In this case, an atomistic approach has been followed based on the analogy with noble gas solids and colloidal crystals. Also in this case, disordered spatial distribution of the spheres is described statistically. For the case of colloidal aggregates, a total two-body mean-field interaction potential is used which includes the Born repulsion energy. This latter contribution plays an essential role in determining the compression behavior of systems of particles aggregated in the primary minimum of the potential well and, therefore, must not be neglected. Both the expression of the Young's modulus and that of the compressibility modulus derived in this work are found to be consistent with two distinct sets of experimental data which recently appeared in the literature. (C) 2007 American Institute of Physics. [DOI: 10.1063/1.2792995]
\end{abstract}

\section{INTRODUCTION}

Granular materials are found in many fields of technology and are crucial to the understanding of many phenomena in Earth's science. ${ }^{1}$ The modeling of mechanical and, especially, elastic properties of disordered granular materials have stimulated considerable efforts in the physics community in the past decades. ${ }^{2-5}$ In the most studied case, frictionless contact is assumed and insights into the phenomenology have been obtained by means of the force network ensemble approach. ${ }^{3,4}$ Given the typically hyperstatic nature of the problem (coordinates and forces are underdetermined) and the highly spatially inhomogeneous and discrete character of the involved networks of grains, research has been focused mainly on stochastic simulations. However, in many practical situations, the particles constituting the granular material do strongly interact with each other. A specific but very often encountered class of materials is thus represented by ensembles of particles where each one is connected with other particles through interparticle bonds. The latter ones are crucial for the cohesion of the material. Just an example is given by colloidal aggregates where particles are connected by means of colloidal forces. Colloidal aggregates, their formation, ${ }^{6}$ rheology, and shear-induced breakage have been studied extensively also from the point of view of mechanical resistance and response to shear. ${ }^{7-16}$ Most of these works refer to aggregates under fluid shear so that mechanical properties cannot be decoupled from the shear history and the problem of characterizing the elasticity of such aggregates

\footnotetext{
a) Author to whom correspondence should be addressed. Fax: 0041-446321082. Electronic mail: alessio.zaccone@chem.ethz.ch
}

still remains elusive. Therefore, in many cases, empirical relations for the dependence of the shear modulus $G$ on the solid volume fraction $\phi$ of the aggregate of the type ${ }^{13-15} G$ $\sim \phi^{n}$, where $n$ is an empirical exponent, are used which do not contain much information about the underlying physics. An experimental breakthrough has been recently achieved by means of optical tweezers which allow exerting a controlled load on colloidal aggregates so as to probe their elastic response. In this way, it has been possible to directly study the elasticity of the interparticle bond which represents the starting point of our understanding of aggregates elasticity. ${ }^{17,18}$

More progress has been achieved in studying the elastic properties of colloidal crystals. ${ }^{19,20}$ This is mainly due to the important simplifications brought about by the ordered structure of crystals. In particular, it is straightforward to apply the same treatment that proved valid in the past to describe the elasticity of noble gas solids. ${ }^{21}$ The theory is accurate to such an extent that it has been recently used to extract surface charge information from measurements of elastic constants. ${ }^{22}$ In fact, for colloidal crystals, the interparticle interaction is dominated by the electrostatic repulsion (often modeled as a simple Yukawa potential), so that the particles are found to be in a secondary minimum of the potential and the lattice spacing is large, thus, giving rise to the wellknown diffraction phenomena in the visible light spectrum. However, the application of such a methodology, to our knowledge, has been attempted rarely in the case of disordered packing. ${ }^{19}$

In this work, a theoretical model is developed with the aim of providing analytical formulas for predicting the elastic moduli of aggregates of spheres randomly distributed in 


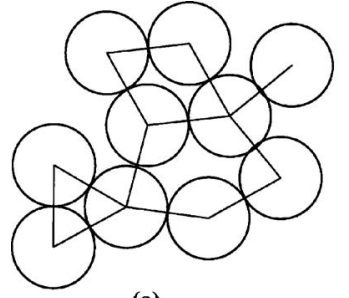

(a)

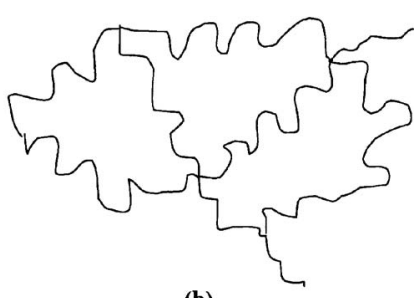

(b)
FIG. 1. (a) Schematic two-dimensional (2D) illustration of a network of bonded particles. The basic elastic elements of the network are the interparticle bonds schematized by means of segments connecting the centers of the spheres. These latter ones constitute the junctions of the network. (b) Schematic of a rubber network. The lines represent polymer chains (the basic elements) and the junctions are given by the cross-link points.

space and connected by cohesive interparticle bonds. Since the concept of random packing is not well defined, ${ }^{23}$ we should point out that the previous definition refers mainly to mesoscopic particulate materials having such disordered structures as they can be found in atomic glasses. Even though it is likely that a certain degree of order is present in these structures, as recently indicated by Truskett and co-workers, ${ }^{23,24}$ we make the reasonable traditional assumption that no trace of long-range order is present. This enables us to use exact models available in the literature to account for the spatial arrangement of the spheres.

The particles are assumed to form a well defined network in space with the bonds being the elementary elastic constituents of the network. Thus, if it is further assumed that the junctions of the network (i.e., the centers of the particles) do not move in space upon deformation (which should be reasonably true for dense enough systems), then a fixedjunction model for the network can be employed. This has been combined with a statistical mechanical description of the nearest-neighbor statistics of the spheres and with a physical description of the interparticle interactions based on surface forces. Contact adhesion, always occurring with locally nonperfectly rigid bodies, was also considered based on recent experimental evidences.

A similar approach has been adopted for the derivation of the bulk modulus. In this case, the well established method of deriving the bulk modulus from the mean-field two-body potential energy of the particles as it is usually done for colloidal crystals ${ }^{19,22}$ has been applied. Again, this is coupled to an appropriate statistical model accounting for the random isotropic distribution of the particles.

\section{YOUNG'S AND SHEAR MODULI OF IDEAL NETWORKS OF SPHERES}

A possible simplified approach to the problem of finding physically meaningful expressions for the elastic moduli of particle aggregates is to view the disordered isotropic aggregate of spheres as a network whose elementary constituents are the interparticle bonds and where the particles are the junctions. This establishes an analogy between the structure of this kind of aggregates and rubber materials where the corresponding basic elastic elements are given by the polymer chains and the junctions are given by the cross-links (Fig. 1). The general approach is to consider the deformation

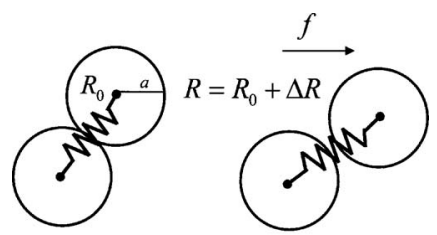

(a)

(b)

FIG. 2. (a) The interparticle bond in the rest (equilibrium) configuration having length $R_{0}$ schematized as a spring joining the centers of the spheres. (b) The interparticle bond in the strained configuration under the action of a force $f$. The interparticle distance is displaced by a displacement $\Delta R$.

free energy of a single interparticle bond, treated as a spring, and then derive the stress-strain relation for the network of bonds under the hypothesis of affine transformation. Based on it, when the aggregate is deformed, each individual elastic element (the interparticle bond) moves in proportion to the deformation of the whole sample. This approach is similar to the one used to derive the first statistical mechanical theory of the mechanical properties of polymers, namely, the theory of rubber elasticity. Notice, however, that the thermodynamics of deformation of a polymer chain is essentially different with respect to that of an interparticle bond. In fact, the main contribution to the free energy of a strained polymer chain is entropic, whereas, in the case of an interparticle bond, this is purely elastic and reversible. This becomes evident on writing down the first principle of thermodynamics for an elastic body undergoing isothermal extensional strain for which the internal energy coincides with the Helmholtz free energy $F$. The change of this latter quantity is given by ${ }^{25}$

$$
\mathrm{d} F=-T \mathrm{~d} S+f \mathrm{~d} L
$$

where $f$ denotes the applied force and $L$ is the macroscopic length of the body. In the case of an interparticle bond treated as an elastic spring, the entropy variation can be ignored, so that we can write ${ }^{25} f=(\partial F / \partial R)_{T, V}$, where $R$ is the length of the spring. Recall the definition of single-bond elastic modulus as $K_{0} \equiv \partial f /\left.\partial R\right|_{R=R_{0}}$, where $R_{0}$ is the length of the spring, i.e., of the interparticle bond at rest, which is $R_{0} \approx 2 a$ ( $a$ is the radius of the particles). Differentiating both sides of Eq. (1) with respect to $R$ and introducing the definition of $K_{0}$ lead to $\partial^{2} F /\left.\partial R^{2}\right|_{R=R_{0}}=K_{0}$. Assuming that the relation between $f$ and $R$ is linear (linear elasticity), for a given displacement of the spring $\Delta R$, as schematically represented in Fig. 2, twofold integration of Eq. (1) between the length at rest, $R_{0}$ [Fig. $2(\mathrm{a})]$, and the length of the deformed spring, $R_{0}+\Delta R$ [Fig. $2(b)]$, gives the following second-power law for the difference in free energy that accompanies the deformation of the $i$ th spring:

$$
\Delta F_{i}=K_{0} \Delta R^{2} .
$$

The extension ratio or stretch ratio is defined macroscopically as $^{26} \lambda=\left(L_{0}+\Delta L\right) / L_{0}$, where $L_{0}$ is the macroscopic sample length in the equilibrium position at rest and $\Delta L$ is the macroscopic displacement (see Fig. 3 for the schematic of the deformation of a macroscopic sample of material along the $y$ axis). Let us now refer to a spring in Cartesian space having an extremity fixed at the origin of the axes, whose position in the nondeformed state is given by the vec- 


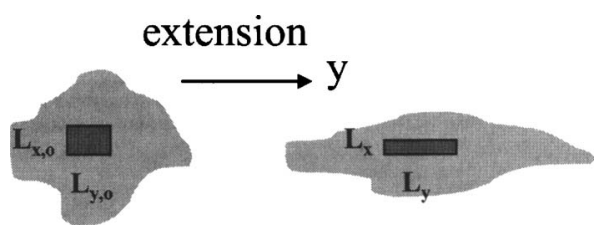

FIG. 3. Macroscopic extensional deformation of the elastic body along the $y$ direction in 2D. The deformation of the small rectangular macroscopic element implies stretching along the $y$ direction and compression along the $x$ direction. This can be described in terms of orthogonal coordinate transformation by introducing the stretch ratio $\lambda$ (see the text).

tor $\mathbf{R}_{0}=\left(R_{x, 0}, R_{y, 0}, R_{z, 0}\right)$ as schematized in Fig. 4. Based on the hypothesis of affine deformation, in the deformed state the length of the spring transforms according to the following coordinate transformation law: ${ }^{26}$

$$
\mathbf{R}=\left(\begin{array}{l}
R_{x}=\lambda_{x} R_{x, 0} \\
R_{y}=\lambda_{y} R_{y, 0} \\
R_{z}=\lambda_{z} R_{z, 0}
\end{array}\right) .
$$

We can now write the square of the displacement in terms of the position of the spring prior to deformation as follows:

$$
\begin{aligned}
\Delta R^{2}=|\Delta \mathbf{R}|^{2}= & \left|\mathbf{R}-\mathbf{R}_{0}\right|^{2}=\left(R_{x}-R_{x, 0}\right)^{2}+\left(R_{y}-R_{y, 0}\right)^{2} \\
& +\left(R_{z}-R_{z, 0}\right)^{2} .
\end{aligned}
$$

For $R_{x, 0}^{2}=R_{y, 0}^{2}=R_{z, 0}^{2}=R_{0}^{2} / 3$, Eq. (4) reduces to

$$
\Delta R^{2}=|\Delta \mathbf{R}|^{2}=\frac{R_{0}^{2}}{3}\left[\left(\lambda_{x}^{2}+\lambda_{y}^{2}+\lambda_{z}^{2}\right)-2\left(\lambda_{x}+\lambda_{y}+\lambda_{z}\right)+3\right] .
$$

In the case of uniaxial extension along the $y$ axis and for an incompressible material, we have $\lambda_{y}=\lambda, \lambda_{x}=\lambda_{z}=\lambda^{-1 / 2}$, and $\lambda_{x} \lambda_{y} \lambda_{z}=1$. Hence, Eq. (5) becomes

$$
\Delta R^{2}=\frac{R_{0}^{2}}{3}\left[\left(\lambda^{2}+\frac{2}{\lambda}\right)-2\left(\lambda+\frac{2}{\sqrt{\lambda}}\right)+3\right] .
$$

We now have a manageable expression for the free energy of deformation of an interparticle bond,

$$
\Delta F_{i}=K_{0} \frac{R_{0}^{2}}{3}\left[\left(\lambda^{2}+\frac{2}{\lambda}\right)-2\left(\lambda+\frac{2}{\sqrt{\lambda}}\right)+3\right] .
$$

To obtain the total free energy of the aggregate, we extend the summation to all bonds/springs by introducing the density of bonds $\langle N\rangle=(1 / 2)\left(n \phi /\left(4 / 3 \pi a^{3}\right)\right)$, where $n$ is the mean number of bonds per particle and $\phi$ is the solid volume frac-

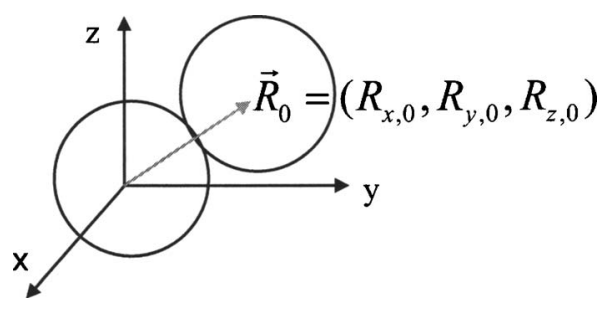

FIG. 4. The fixed-junction model. To analyze the deformation of a single bond, the center of one particle is treated as fixed at the orthogonal axes' origin and the vector $\bar{R}_{0}$ joining the centers of the spheres is given by three components in Cartesian space. tion. The factor $1 / 2$ arises in order to avoid counting the interparticle bonds twice.

The $n$ value can be evaluated upon introduction of a radial pair correlation function evaluated at particle-particle contact, i.e., at a center-to-center distance equal to $R_{0} \approx 2 a$. Torquato et al. derived the nearest-neighbor statistical descriptors for the microstructure of random isotropic packings of hard spheres. ${ }^{27,28}$ In particular, Torquato ${ }^{28}$ derived expressions for the radial distribution function as a function of the volume fraction on the basis of the well-known CarnahanStarling approximation for equilibrium hard spheres. The derived expressions have been proposed to apply to the modeling of nonequilibrium disordered packings studied in materials science, including amorphous solids and composite particulates. $^{28}$ More recently, it has been demonstrated by means of an order metrics approach ${ }^{23,24}$ that the nonequilibrium (glassy) systems may have various degrees of increased order with respect to equilibrium systems, although the former was traditionally assumed to share the same spatial structure as the latter. An important outcome is that the traditionally accepted limit of random close packing, which can be better termed as maximally random jammed state, ${ }^{23}$ is not unique and cannot be defined unambiguously. In the same way, no unique "random packing" can be defined but a variety of disordered packings with different degrees of order and upper limiting densities can be created which depend on the protocol (i.e., the process history). However, statistical structural descriptors accounting for these important findings are still missing in the literature. Therefore, in the following, the traditional equilibrium hard-sphere approach introduced by Torquato $^{28}$ has been adopted and should be regarded as an approximation of the real structure. We believe that such approximation still can correctly describe the realistic scaling of the computed properties with the volume fraction. Furthermore, the hard-sphere model is known to also provide an excellent approximation of those systems where dispersive attractions sum up with the hard-sphere repulsion such as the Lennard-Jones system, as stated by the perturbation theory ${ }^{29}$ and recently confirmed by simulations. ${ }^{30}$ The expression derived by Torquato for the contact-value radial distribution function in disordered packings of hard spheres is given by ${ }^{28}$

$$
n \equiv g\left(R_{0}\right)=\frac{(1-\phi / 2)}{(1-\phi)^{3}}
$$

Upon neglecting fluctuations of the network's junctions, we can now run a summation over all the contributions coming from all the bonds in the unit volume. This approach is quite straightforward but it is expected to work reasonably well for dense materials where each particle is bonded to a relatively high number of nearest neighbors, thus, remaining constrained to a nearly fixed position in space. Deviations from this picture are expected for less dense materials where the particles are bound to a smaller number of neighbors and are therefore free to fluctuate in space. Hence, one can obtain the difference in free energy associated to deformation per unit volume as 


$$
\begin{aligned}
\Delta \widetilde{F}_{\text {tot }}=\frac{1}{V} \sum_{i} \Delta F_{i}= & \langle N\rangle K_{0} \frac{R_{0}^{2}}{3}\left[\left(\lambda^{2}+\frac{2}{\lambda}\right)-2\left(\lambda+\frac{2}{\sqrt{\lambda}}\right)+3\right] \\
= & \frac{K_{0} R_{0}^{2}}{8 \pi a^{3}} \phi \frac{(1-\phi / 2)}{(1-\phi)^{3}}\left[\left(\lambda^{2}+\frac{2}{\lambda}\right)\right. \\
& \left.-2\left(\lambda+\frac{2}{\sqrt{\lambda}}\right)+3\right] .
\end{aligned}
$$

In the regime of linear (Hookean) elasticity, the stretch ratio $\lambda$ can be expressed as a function of the strain $e_{y y}$ as $\lambda=1$ $+e_{y y}{ }^{26}$ Upon substituting this into Eq. (9), we obtain a relation between the applied stress and the strain $e_{y y}$,

$$
\begin{aligned}
\Delta \widetilde{F}_{\text {tot }}= & \frac{K_{0} R_{0}^{2}}{8 \pi a^{3}} \phi \frac{(1-\phi / 2)}{(1-\phi)^{3}}\left[\left(\left(1+e_{y y}\right)^{2}+\frac{2}{\left(1+e_{y y}\right)}\right)\right. \\
& \left.-2\left((1+e)+\frac{2}{\sqrt{\left(1+e_{y y}\right)}}\right)+3\right] .
\end{aligned}
$$

Recall that the free energy per unit volume of a deformed anisotropic body is $\widetilde{F}=(1 / 2) C_{i k l m} e_{i k} e_{l m}$, where $C_{i k l m}$ is the rank-4 tensor of the elastic constants. ${ }^{31}$ We can now apply the fundamental relation of deformation thermodynamics, based on which the associated stress is given by the derivative of the free energy of deformation per unit volume with respect to the strain, ${ }^{31} \sigma_{i k}=\left(\partial \tilde{F} / \partial e_{i k}\right)_{T}=C_{i k l m} e_{l m}$. For the case of an isotropic material and uniaxial extension along the $y$ axis, the tensor formulation reduces to a scalar equation and we arrive at the following stress-strain relation:

$$
\begin{aligned}
\sigma_{y y}=\frac{\partial \Delta \widetilde{F}_{\text {tot }}}{\partial e_{y y}}= & \frac{K_{0} R_{0}^{2}}{8 \pi a^{3}} \phi \frac{(1-\phi / 2)}{(1-\phi)^{3}}\left[2\left(1+e_{y y}\right)-\frac{2}{\left(1+e_{y y}\right)^{2}}\right. \\
& \left.-2\left(1-\frac{1}{\left(1+e_{y y}\right)^{3 / 2}}\right)\right] .
\end{aligned}
$$

This relation is nonlinear (i.e., non-Hookean) but it can be linearized in the limit of small strains through a Maclaurin expansion which yields the following linearly elastic relationship:

$$
\sigma_{y y}=\left[\frac{3 K_{0} R_{0}^{2}}{8 \pi a^{3}} \phi \frac{(1-\phi / 2)}{(1-\phi)^{3}}\right] e_{y y} .
$$

Thus, one can conclude that the Young's modulus of the network is given by

$$
E=\frac{3 K_{0} R_{0}^{2}}{8 \pi a^{3}} \phi \frac{(1-\phi / 2)}{(1-\phi)^{3}}=\frac{3 K_{0}}{2 \pi a} \phi \frac{(1-\phi / 2)}{(1-\phi)^{3}} .
$$

Under the assumption of incompressibility, which is quite reasonable for elastic networks, one can assume the Poisson's ratio to be $\nu=0.5$. Then, from the theory of elasticity, the shear modulus follows as

$$
G=E / 3=\frac{K_{0} R_{0}^{2}}{8 \pi a^{3}} \phi \frac{(1-\phi / 2)}{(1-\phi)^{3}}=\frac{K_{0}}{2 \pi a} \phi \frac{(1-\phi / 2)}{(1-\phi)^{3}} .
$$

In order to evaluate the above expressions for the elastic moduli of a random ensemble of particles connected by bonds, it is necessary to consider the nature of the interparticle bonds in order to describe the single-bond elasticity in a suitable way. This is done in the following section.

\section{SINGLE-BOND ELASTICITY: CONSTITUTIVE RELATIONS}

\section{A. Perfectly rigid colloidal particles (nonadhesive case)}

Following the classic description of colloidal forces, the interaction between two perfectly rigid spherical particles is formulated in terms of two-body interparticle potential energy with central symmetry. The interaction potential, in turn, is associated with a central interaction force between the particles which is responsible for the interparticle bond strength between them. The Derjaguin-Landau-VerweyOverbeek (DLVO) theory states that the mean-field interparticle potential is made up of two contributions, namely, the attractive van der Waals potential and the repulsive electrostatic double layer (EDL) potential. ${ }^{32}$ However, the van der Waals forces alone would lead to an asymptotically decreasing potential at short interparticle distances which is not realistic. To account for short-range repulsion which ultimately arises due to the overlap of atomic shells (Pauli's exclusion principle), the Born energy contribution is inserted with the formulation made by Feke et al. ${ }^{33}$ who derived manageable formulas by using the Hamaker summation approach. Hence: $U_{\mathrm{TOT}}=U_{\mathrm{vdW}}+U_{\mathrm{EDL}}+U_{\mathrm{Born}}$. Assuming the general Hamaker form for the van der Waals potential between two spheres, the Hogg-Healy-Fuerstenau ${ }^{34}$ approximation for the repulsive EDL interaction and the expression of the Born repulsion energy suggested by Feke et al., ${ }^{33}$ leads to the following total interparticle potential energy:

$$
\begin{aligned}
U_{\mathrm{TOT}}(r)= & -\frac{A_{H}}{6}\left[\frac{2}{r^{2}-4}+2 r^{2}+\ln \frac{r^{2}-4}{r^{2}}\right] \\
& +N_{12} \frac{1}{r}\left[\frac{r^{2}-14 r+54}{(r-2)^{7}}+\frac{-2 r^{2}+60}{r^{7}}\right. \\
& \left.+\frac{r^{2}+14 r+54}{(r+2)^{7}}\right]+\frac{4 \pi \varepsilon_{0} \varepsilon_{r} a \psi_{0}^{2}}{r} \ln \{1+\exp [ \\
& -\kappa a(r-2)]\},
\end{aligned}
$$

where $A_{H}$ is the Hamaker constant, $r$ is the interparticle center-to-center distance normalized by the particle radius $a$, $N_{12}$ is a parameter expressing the strength of the van der Waals attraction relative to the Born repulsion. Furthermore, $\psi_{0}$ is the surface electric potential and $\kappa$ is the Debye-Hückel parameter. $N_{12}$ is defined as $N_{12}=4(\sigma / a)^{6}(4 ! / 10 !)$, where $\sigma$ is the atomic collision diameter. ${ }^{33}$ In practice, however, when computing the force acting between two neutral rigid spheres at contact, only the van der Waals term is considered. ${ }^{35}$ The single-bond spring constant for the case of perfectly rigid (i.e., nonadhering) neutral spheres can be simply given by

$$
K_{0, \mathrm{NA}} \equiv \frac{(-\partial U / \partial R)}{R_{0}}=\frac{f}{R_{0}}=\frac{2 \pi a \gamma_{\mathrm{SL}}}{R_{0}} \approx \pi \gamma_{\mathrm{SL}}
$$

where, for simplicity, the Derjaguin approximation has been used and linear elasticity has been assumed. The quantity $\gamma_{\mathrm{SL}}$ 
is the solid-liquid surface energy. One can now insert this constitutive equation into Eq. (13), which yields the following expression for the elastic Young's modulus:

$$
E_{\mathrm{NA}}=\frac{3}{2} \phi \frac{(1-\phi / 2)}{(1-\phi)^{3}} \frac{\gamma_{\mathrm{SL}}}{a} .
$$

It is seen that the Young's modulus depends on the solid volume fraction of the material, $\phi$, the radius of the particles, $a$, and the surface energy of the solid-liquid interface, $\gamma_{\mathrm{SL}}$. Very similar expressions of the interparticle force are available for the case of particles in vapor and in vacuum (just by replacing $\gamma_{\mathrm{SL}}$ with the corresponding values for the solidvapor and solid-vacuum interfaces, $\gamma_{\mathrm{SV}}$ and $\gamma_{S}$, respectively) so that the present analysis can be easily extended to packing of whatever system of rigid spheres.

\section{B. Particles deformable at contact (adhesive case)}

The case discussed in the previous subsection is somehow ideal since real particles are never completely rigid but can undergo elastic deformation upon contact. This, in the case of spheres, gives rise to a nonzero circular contact area, with a radius denoted by $a_{c}$, which is associated with attractive van der Waals forces between the two surfaces. ${ }^{35}$ Recent experimental work done by Pantina and Furst ${ }^{17,18}$ has demonstrated that, in the case of colloidal polymethyl metacrylate (PMMA) particles, the contact mechanics can be described conveniently by means of the Johnson-KendallRoberts (JKR) theory. ${ }^{36}$ These authors studied the response of linear chains of aggregated PMMA particles by means of optical tweezers and were able to measure the spring constant of the colloidal bonds. The experimental values of $K_{0}$ were found in very good agreement with values calculated with the following expression: ${ }^{17} K_{0}=3 \pi a_{c}^{4} E_{m} / 4 a^{3} . K_{0}$ is proportional to the area moment of inertia of the cylindrical neck in the region between the particles, $I=\pi a_{c}^{4} / 4 . E_{m}$ is the Young's modulus of the bulk material and the radius of the contact area, $a_{c}$, is given according to the JKR theory as ${ }^{17,35}$ $a_{c}=\left(3 \pi a^{2} W_{\mathrm{SL}} / 2 B_{m}\right)^{1 / 3}$, where $W_{\mathrm{SL}}$ is the adhesion energy (a function of the intersurface forces) and $B_{m}$ is the compressibility or bulk modulus of the material that constitutes the particles. $a_{c}$ is measured, for micron particles, with optical techniques. Inserting $K_{0}=3 \pi a_{c}^{4} E_{m} / 4 a^{3}$ into Eq. (14) yields the following form of the Young's modulus in the case of contact adhesion:

$$
E_{A}=\frac{9}{8} E_{m} \phi \frac{(1-\phi / 2)}{(1-\phi)^{3}}\left(\frac{a_{c}}{a}\right)^{4} .
$$

The Young's modulus as given by Eq. (17) for the case of perfectly rigid spheres and by Eq. (18) for adhering spheres is plotted as a function of the solid volume fraction in Fig. 5, for a system of uncharged PMMA particles $(1.5 \mu$ in diameter). $\gamma_{\mathrm{SL}}=0.19 \mathrm{~J} / \mathrm{m}^{2}$ is the surface energy of the PMMAwater interface. ${ }^{18}$ The interparticle bond spring constant has been taken equal to $0.8 \mathrm{~N} / \mathrm{m}$ as predicted by the JKR theory for this system. ${ }^{17}$ However, Pantina and Furst ${ }^{18}$ found that the JKR theory leads to an overestimation of the experimental values if one does not account for the electrostatics in a proper way. They found a significant improvement by incor-

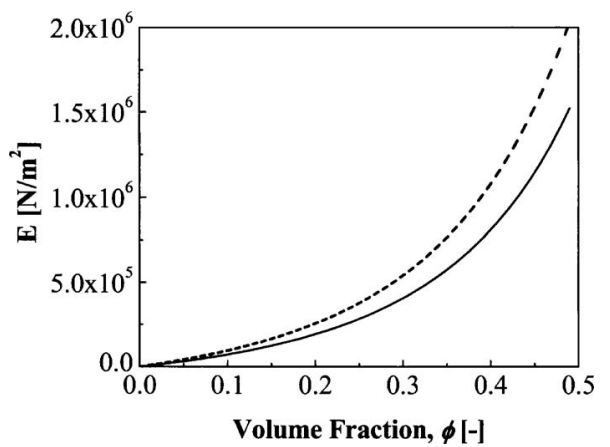

FIG. 5. The Young's modulus as a function of the solid volume fraction calculated from Eqs. (17) and (18) for the case of perfectly rigid spheres (continuous line) and adhering spheres (dashed line), respectively.

porating electrostatic and even ion-correlation effects into the adhesion energy $W_{\mathrm{SL}} \cdot{ }^{18}$ Another possible contribution to this systematic overestimation might be the well-known fact that the JKR theory ignores the roughness of the particle surface. It has been shown recently ${ }^{37}$ that, in the case of polystyrene particles, the surface roughness plays a significant role in the adhesion mechanism and leads to a decreased adhesion with respect to that predicted for perfectly smooth surfaces. This eventually leads to a "decoration" in the formulation of the JKR relations. ${ }^{37}$

\section{COMPRESSIBILITY (BULK) MODULUS}

In the hydrostatic compression of a solid body, the bulk modulus is defined as the coefficient relating the hydrostatic pressure $p$ to the trace of the strain tensor. Under the assumption of small deformations, the following relation is valid: $B=-(1 / V)(\partial p / \partial V)_{T}$, where $V$ is the volume of the sample. The pressure acting on an aggregate of particles is equilibrated by an equal and opposed pressure originated by the energy of the interparticle bonds. Hence, the pressure can be expressed, also in the case of macroscopic spheres, as $p$ $=-\mathrm{d} U / \mathrm{d} V$. We can, thus, write the bulk modulus in terms of particle-averaged quantities, $B=v(\partial / \partial v)(\partial u / \partial v)$, where $v$ $=V / N$ is the volume per particle in the packing. In order to compute the interaction energy per particle, $u$, rigorously one should first consider the interaction of the particle chosen at the origin with all the particles in the sample, $\sum_{R \neq \mathbf{0}} U(\mathbf{R})$, where $\mathbf{R}$ is the vector connecting the center of the particle at the origin with the centers of all the other particles in the network. If this quantity is multiplied by the number of particles in the sample, however, what one gets is twice the total potential energy of the sample. Therefore, the interaction energy per particle must be given by $u=(1 / 2) \Sigma_{\mathbf{R} \neq \mathbf{0}} U(\mathbf{R}){ }^{38}$ Assuming that the interaction energy rapidly decays after the first coordination shell, the previous quantity can be conveniently expressed by limiting the summation to the nearest neighbors and introducing, once again, the radial pair correlation function at contact, so that the previous expression simplifies to 


$$
u=\frac{1}{2} \sum_{N N} U(R)=\frac{1}{2} n U(R)=\frac{1}{2} \frac{(1-\phi / 2)}{(1-\phi)^{3}} U(R),
$$

where the sum is over the $n$ nearest-neighbors particles, i.e., over the particles bonded to the particle chosen at the origin. It is now required to find a suitable expression for the volume per particle in the random packing, $v$. To this aim, the simplest approach is to use the analytical result originally derived by Hertz, based on which, in an ensemble of Poisson distributed particles, $v=(R / 0.55396)^{3}$, where $R$ is the interparticle distance. ${ }^{39}$ It follows that $\partial / \partial v$ $=\left[(0.55396)^{3} / 2 R^{2}\right] \partial / \partial R$. Thus, the bulk modulus can be written as $B=\left[(0.55396)^{3} / 9\right] R(\partial / \partial R)\left(1 / R^{2}\right)(\partial / \partial R) u$. We are interested in evaluating the bulk modulus at the equilibrium interparticle distance, i.e., at the location where $\partial u / \partial R \rightarrow 0 .^{38}$ Inserting Eq. (19) into the above $B$ expression gives the bulk modulus as follows:

$$
\begin{aligned}
B_{0} & =\left.\frac{(0.55396)^{3}}{9} \frac{1}{R_{0}} \frac{\partial^{2} u}{\partial R^{2}}\right|_{R=R_{0}} \\
& =\left.\frac{(0.55396)^{3}}{18} \frac{(1-\phi / 2)}{(1-\phi)^{3}} \frac{1}{R_{0}} \frac{\partial^{2} U}{\partial R^{2}}\right|_{R=R_{0}} .
\end{aligned}
$$

Equation (20) provides an exact formula for the compressibility or bulk modulus of an isotropic random ensemble of spheres, as a function of the mean-field potential energy of interaction between nearest-neighbor particles and of the solid volume fraction. Also in this case, as in the cases of the Young's and shear moduli, a statistical description of the spatial disordered distribution of the spheres is combined with a physical description of the interparticle bond. This derivation does not account for contact adhesion effects and, rigorously, can be applied only to perfectly rigid spheres. It is worth noticing that, in the general case of the bulk modulus of charged colloidal aggregates, the total interparticle potential, as given by Eq. (15), is required, which cannot reduce to the van der Waals term alone. In fact, in this case, since the bulk modulus is related to the resistance of the material to hydrostatic pressure, it is evident that the Born repulsion plays an essential role in determining such kind of behavior and, therefore, must be accounted for in calculating the bulk modulus.

\section{DISCUSSION AND COMPARISON WITH EXPERIMENTAL DATA}

In the previous parts, simple exact relationships expressing the elastic moduli of randomly distributed aggregated spheres have been derived starting from thermodynamic first principles and upon application of the linear elasticity theory. Physically, relevant and measurable parameters entering the theoretical formulation are the solid volume fraction, the primary particles size, the interparticle interaction parameters, and the contact mechanics parameters (the latter ones including the contact radius and the Young's modulus of the bulk material). Particularly relevant to applications, from materials design to geophysical research, is the dependence of the elastic moduli on the solid volume fraction (or porosity) of the material sample. Relations (17) and (18) express the Young's modulus as a function of physical parameters that are either known a priori or experimentally measurable. Shahidzadeh-Bonn et al $^{40}$ experimentally studied the dependence of the Young's modulus on the porosity (and thereof on the solid volume fraction $\phi$ ) of a disordered packing constituted by glass beads, $a \approx 57 \mu \mathrm{m}$, that were sintered in order to induce softening and adhesion between the spheres. The size of the particles situates the system out of the colloidal domain and the particle-particle interaction can be at best understood in terms of contact mechanics as explained in Section IV.B. The relevant quantity in this regard is, thus, the contact radius of the cylindrical neck between the particles which was measured and found to be $a_{c}=14 \mu \mathrm{m}$. This system appears to be a suitable ground to test the theory presented here. These authors compared their results with analytical predictions of the so-called self-consistent (SC) method. ${ }^{41,42}$ This latter one was developed for calculating properties of heterogeneous materials based on the principle that the perturbations brought about by the inclusions (cavities, in this case) must be zero on average. The SC model for porous materials used by these authors reads

$$
E=E_{\mathrm{mat}}\left[\frac{20 \nu_{\mathrm{mat}}+63 \varepsilon^{2} \nu_{\mathrm{mat}}-69 \varepsilon \nu_{\mathrm{mat}}-27 \varepsilon^{2}+15 \varepsilon+8+(3 \varepsilon-4) \sqrt{\Delta}}{10\left(2 \nu_{0}-1\right)\left(3 \varepsilon \nu_{\mathrm{mat}}-3 \varepsilon+2\right)}\right]
$$

where $\varepsilon$ is the porosity $(\varepsilon=1-\phi), E_{\text {mat }}$ is the Young's modulus of the bulk material, $\nu_{\text {mat }}$ is the Poisson's ratio of the bulk material, and $\Delta$ is a second order polynomial function of $\varepsilon$ and $\nu_{0}$. A common shortcoming of the SC model, despite its wide use, is that it ignores specific microstructural features of the material such as morphology and spatial organization. ${ }^{41}$ In Fig. 6, the model proposed in this work is compared with the experimental results reported by Shahidzadeh-Bonn et $a l .,{ }^{40}$ as well as with the prediction of the SC model. The parameters for the computation of the theoretical curves have been chosen consistently with the parameters relative to the experimental system. The Young's modulus for glass is ${ }^{43}$ $E_{\text {mat }}=9.4 \times 10^{-10} \mathrm{~N} / \mathrm{m}^{2}$. A Poisson's ratio of 0.3 for glass has been used. The contact radius is the one measured and reported by these authors, namely, $a_{c}=14 \mu \mathrm{m}$. The authors show a fitting of the experiments by means of Eq. (21) where a good accord is achieved by using $E_{\text {mat }}$ as a fitting parameter, $E_{\text {mat }}=2.2 \times 10^{-10} \mathrm{~N} / \mathrm{m}^{2}$. This value is of the same order 


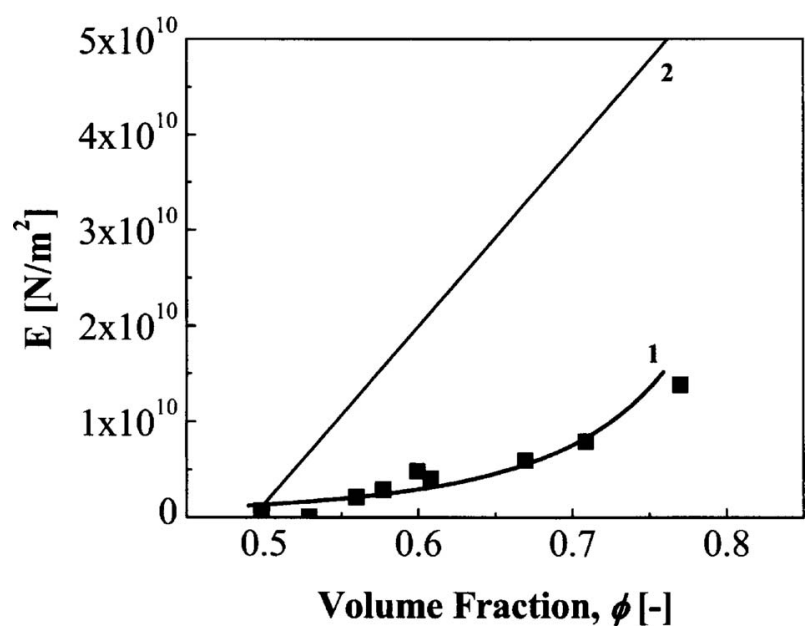

FIG. 6. The Young's modulus as a function of the solid volume fraction Experimental data from Ref. 35 (squares) are compared with theoretical predictions from Eq. (18) (line 1) and with predictions from the SC approximation, Eq. (21) (line 2). Physical parameters of the experiments used for the theoretical calculation are $a=57 \mu \mathrm{m}, \quad a_{c}=14 \mu \mathrm{m}, \quad E_{\text {mat }}=9.4$ $\times 10^{-10} \mathrm{~N} / \mathrm{m}^{2}$, and $\nu_{\mathrm{mat}}=0.3$.

of magnitude of the typical value for glass but probably still too low (indeed about four times lower). In Fig. 6, both our model and the SC model are calculated with the value usually employed for silica glass and reported above.

As can be seen, the SC model, if no adjustable parameter is used, is unable to predict the experimental results, except for the point at the lowest $\phi$, and it fails also in reproducing the trend of the experimental curve qualitatively. In particular, the SC model systematically overestimates the experimental data and especially the last point is overestimated nearly by a factor of 5 . Instead, the model presented within this work [Eq. (18)] is found to be consistent with the experimental data in the entire range of the experimental investigation. One reason that could explain the good consistency may be that the experiments refer to a rather dense system of particles which are strongly bonded to each other. This means that nonideal effects in the network's deformation such as motion and rearrangement of the particles in the network, which are ignored by the model, may in this case be of small extent, due to the compact nature of the network. This hypothesis is further corroborated by what was reported by Pantina and Furst ${ }^{17,18}$ who, even in the case of linearchain aggregates of particles, observed sliding and rolling of the particles past each other only to a small extent. Considered the much higher compactness of the system discussed here, it is reasonable to assume that the particles are not much free to move, and, during the elastic deformation, the network behaves as nearly ideal.

On turning now our attention to the validation of the model for the compressibility modulus, experimental results of compression strength reported by Tang et al. ${ }^{44}$ are compared to theoretical predictions given by Eq. (20). In the experiments, colloidal aggregates of polystyrene spheres (having radius $a=0.57 \mu \mathrm{m}$ ) were obtained under RLCA conditions and then squeezed between parallel plates. The aggregates were produced according to the procedure described in Tang et al., ${ }^{45}$ where an optical micrograph of a typical RLCA

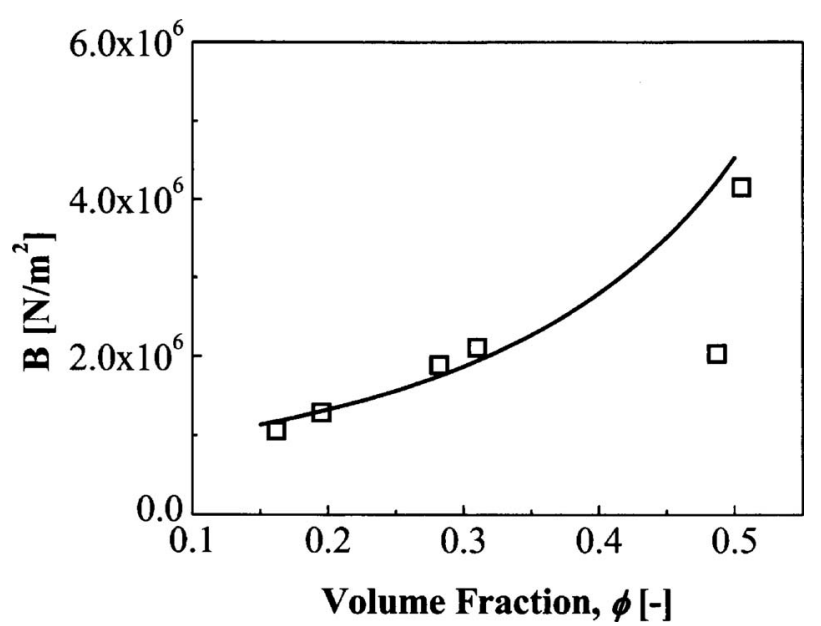

FIG. 7. The bulk modulus calculated based on Eq. (20) with the total meanfield interaction potential given by Eq. (15) (solid line) compared with experimental data reported in Ref. 39 (squares). The total interaction potential has been calculated with the following parameters: $\psi_{0}$ $\approx-20 \mathrm{mV}, \kappa^{-1} \approx 0.68 \mathrm{~nm}, A_{H}=4.12 \times 10^{-21} \mathrm{~J}$, and $\sigma \approx 4 \AA$.

aggregate is also shown, indicating a rather compact structure where each particle has multiple contacts with other particles. The rupture of the aggregates under compression was optically monitored and the applied force was measured. The latter, when divided by the cross-sectional area of the aggregates, gives a measure of the compression strength of the aggregates, which can be regarded as a measure of the compressibility modulus of the aggregates. Tang et al. ${ }^{44}$ fitted these data with a semiempirical model for the tensile strength of aggregates.

One could question the adoption of a correlation function for random packing of hard spheres to model fractal aggregates such as RLCA aggregates. However, one should also bear in mind that the power-law scaling in the pair correlation function typical of fractal aggregates sets in at a distance from the particle which is normally about four times the particle radius. ${ }^{46,47}$ Here, we are interested in the correlation function at contact only (i.e., at the local particle scale), which still can be modeled in a good approximation with the simple correlation used for disordered packing of spheres. The number of bonds per particle within an aggregate predicted from Monte Carlo simulations and simulations of random packing of spheres ${ }^{48,52}$ increases as the solid volume fraction increases. Then, the compressibility modulus also increases with the solid volume fraction, as it is observed experimentally.

The calculated bulk modulus is plotted in Fig. 7 together with the experimental results. The total interparticle potential given by Eq. (15) has been computed with the experimental parameters reported by Tang et al., ${ }^{44}$ i.e., $\psi_{0} \approx-20 \mathrm{mV}$ taken equal to the measured zeta potential, the Debye length $\kappa^{-1}$ $\approx 0.68 \mathrm{~nm}$ corresponding to the ionic strength conditions employed, and the Hamaker constant $A_{H}=4.12 \times 10^{-21} \mathrm{~J}$ as used by the authors. Furthermore, the atomic collision diameter required to compute the Born repulsion term has been taken equal to $\sigma \approx 4 \AA$, which is the value generally used for the carbon atom. The resulting two-particle mean-field potential energy is shown in Fig. 8. There is an energy barrier 


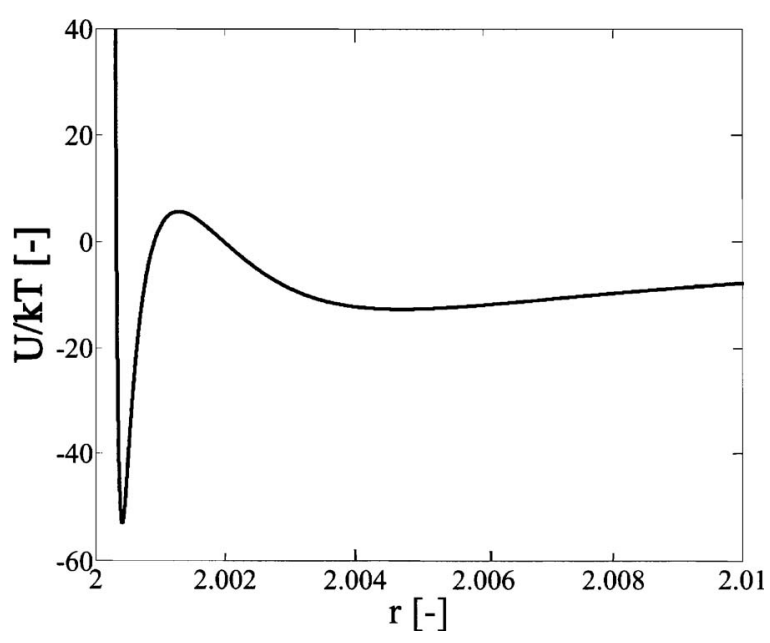

FIG. 8. Mean-field interaction energy curve computed using Eq. (15) for the experimental system of RLCA aggregates reported in Ref. 39. Physical parameters used are $\psi_{0} \approx-20 \mathrm{mV}, \kappa^{-1} \approx 0.68 \mathrm{~nm}, A_{H}=4.12 \times 10^{-21} \mathrm{~J}$, and $\sigma$ $\approx 4 \AA$.

due to the partially unscreened EDL repulsion and a smooth secondary minimum. However, the height of the barrier is of a few $k T$ 's, where $k$ is Boltzmann's constant, so that we can assume that the particles are aggregated in the primary minimum, which occurs at a normalized interparticle distance of $r=2.0004$. The average number of particles per bonds, $n$, as it is approximated by the pair correlation function for random packing, ranges from a value of about 2 in the lower range of $\phi$ up to a maximum value of 6 at the highest volume fraction.

When one considers that no adjustable parameter is used, the agreement between the theory and the experiments is very satisfactory. Apart from a point which is clearly not aligned with the others, the theoretical curve reproduces the experimental data not only qualitatively but also quantitatively. From a quantitative point of view, the interparticle potential chosen (DLVO plus Born energy) turns out to be the correct way of accounting for colloidal interactions, and including the short-range repulsive Born term is necessary to describe the interaction realistically. This is done in analogy with the theory of bulk modulus of solid noble gases where a 6-12 Lennard-Jones potential generally leads to excellent agreement with experiments (with the exception of helium). ${ }^{21,38}$ The present case can then be regarded as an extension of that theory to random ensembles of aggregated colloidal particles.

\section{CONCLUDING REMARKS}

A theoretical model has been developed, starting from thermodynamic first principles, for the elastic moduli of materials constituted by randomly distributed spheres connected by physical interparticle bonds. Granular materials are currently being the object of intense theoretical investigation ${ }^{49}$ but most of the research focuses on packing of frictionless nonbonded particles. The present work instead treats the case of packing of particles that are strongly interconnected, thus constituting a well defined isotropic random network in space. This class of materials is of high relevance for chemi- cal applications (e.g., colloidal aggregates and coagulation processes) as well as for geophysical science ${ }^{50}$ and for the understanding of fracture phenomena in construction engineering (e.g., porous materials). ${ }^{51}$ It is assumed that the strong physical interparticle bonds can be identified as the elementary constituents of a network, and they are modeled as linearly elastic springs whose position remains fixed in space during strain. The deformation of such an ideal network was analyzed by means of the affine transformation hypothesis. The free energy of deformation of the network is consequently expressed through a summation over all the elastic springs in the network, and the disordered distribution of the particles is accounted for by means of the radial pair correlation function for disordered packing of spheres. This introduces a physically meaningful dependence on the solid volume fraction of the material.

Elastic Young's and shear moduli are derived as a function of the single-bond spring elastic constant. Analytical expressions for the latter have been derived by means of constitutive relations for the two distinct cases of perfectly rigid particles (no adhesion) and adhering particles. For the former, the only attractive part of the mean-field interparticle potential is used, as it is usually done, and, for the latter, the JKR theory of adhesion is introduced. As expected, the elastic moduli are slightly larger in the case of adhesion than in the case of perfectly rigid spheres.

An expression for the bulk or compressibility modulus is also derived following a similar approach and adopting a procedure used for noble gas solids and colloidal crystals. In describing the spatial distribution of the spheres, the simple law of distribution of the nearest neighbor in a random ensemble of spheres has been employed, thus replacing the treatment based on lattice constants usually adopted with colloidal crystals. The form of the mean-field interparticle potential is also different, and it includes the van der Waals and EDL terms, as well as the Born repulsion energy which is important to describe the interaction of aggregates of spheres realistically. The Born repulsion term is not crucial in the case of colloidal crystals where the interaction is dominated by the electrostatics but it is of fundamental importance in the case of colloidal particles aggregated in the primary minimum.

Predictions of the theory are compared with recent experimental data available in the literature, and with no adjustable parameters, the agreement is very satisfactory for the Young's modulus of dense packing of glass beads by using the bond spring constant based on contact mechanics. The good agreement, in spite of assuming an ideal network, can be explained by the fact that, in the limit of dense packing, the spheres have multiple contacts with neighboring particles, thus minimizing nonideal network effects such as sliding and rolling of the particles.

Very good agreement is also obtained between our theoretical predictions and literature experimental data for the compressibility or bulk modulus of colloidal aggregates of PS spheres. In this case, it turns our that including the Born short-range repulsion in the total interaction potential is crucial in describing the physics of particle-particle interaction 
in a realistic manner. The bulk modulus is therefore directly determined by interactions at the particle-particle level.

In sum, our analysis, consistent with experimental observations in the literature, suggests that the elastic properties of a particular but ubiquitous category of granular materials, namely, disordered packing of particles connected by physical bonds, may be understood in close relation to the nature and features of the interparticle bonds that seem to play a major role in determining the cohesion and the mechanical behavior of such materials.

\section{ACKNOWLEDGMENTS}

Dr. U. A. Handge at the Institute for Polymers, Department of Materials, ETH Zurich, is acknowledged for his critical comments. Financial support from the Swiss National Science Foundation (Grant No. 200020-113805/1) is gratefully acknowledged.

${ }^{1}$ P. G. de Gennes, Rev. Mod. Phys. 71, S374 (1999).

${ }^{2}$ S. F. Edwards and R. B. S. Oakeshott, Physica A 157, 1080 (1989).

${ }^{3}$ J. H. Snoeijer, T. J. H. Vlugt, W. G. Ellenbroek, M. van Hecke, and J. M. J. van Leeuwen, Phys. Rev. E 70, 061306 (2004).

${ }^{4}$ J. H. Snoeijer, W. G. Ellenbroek, T. J. H. Vlugt, and M. van Hecke, Phys. Rev. Lett. 96, 098001 (2006)

${ }^{5}$ S. Ostojic and D. Panja, Phys. Rev. Lett. 97, 208001 (2006).

${ }^{6}$ M. Lattuada, H. Wu, J. Sefcik, and M. Morbidelli, J. Phys. Chem. B 110 6574 (2006)

${ }^{7}$ M. Doi and D. Chen, J. Chem. Phys. 90, 5271 (1989).

${ }^{8}$ D. Chen and M. Doi, J. Chem. Phys. 91, 2656 (1989).

${ }^{9}$ A. A. Potanin, J. Chem. Phys. 96, 9191 (1992).

${ }^{10}$ A. A. Potanin, R. Derooij, D. Vandenende, and J. Mellema, J. Chem. Phys. 102, 5845 (1995)

${ }^{11}$ L. E. Silbert, R. S. Farr, J. R. Melrose, and R. C. Ball, J. Chem. Phys. 111, 4780 (1999).

${ }^{12}$ M. U. Babler, J. Sefcik, M. Morbidelli, and J. Baldyga, Phys. Fluids 18, 013302 (2006).

${ }^{13}$ M. U. Bäbler, M. Morbidelli, and J. Baldyga, J. Fluid Mech. (submitted).

${ }^{14}$ R. C. Sonntag and W. B. Russel, J. Colloid Interface Sci. 113, 399 (1986).

${ }^{15}$ R. C. Sonntag and W. B. Russel, J. Colloid Interface Sci. 115, 378 (1987).

${ }^{16}$ A. H. L. West, J. R. Melrose, and R. C. Ball, Phys. Rev. E 49, 4237 (1994).

${ }^{17}$ J. P. Pantina and E. M. Furst, Phys. Rev. Lett. 94, 138301 (2005).

${ }^{18}$ J. P. Pantina and E. M. Furst, Langmuir 22, 5282 (2006).

${ }^{19}$ H. M. Lindsay and P. M. Chaikin, J. Chem. Phys. 76, 3774 (1982).

${ }^{20}$ S. Alexander, P. M. Chaikin, P. Grant, G. J. Morales, P. Pincus, and D. Hone, J. Chem. Phys. 80, 5776 (1984).

${ }^{21}$ E. R. Dobbs and G. O. Jones, Rep. Prog. Phys. 20, 516 (1957).
${ }^{22}$ L. Shapran, H. J. Schope, and T. Palberg, J. Chem. Phys. 125, 194714 (2006).

${ }^{23}$ S. Torquato, T. M. Truskett, and P. G. Debenedetti, Phys. Rev. Lett. 84, 2064 (2000)

${ }^{24}$ T. M. Truskett, S. Torquato, and P. G. Debenedetti, Phys. Rev. E 62, 993 (2000).

${ }^{25}$ C. Price, Proc. R. Soc. London, Ser. A 351, 331 (1976).

${ }^{26}$ M. Rubinstein and R. H. Colby, Polymer Physics (Oxford University Press, Oxford, 2003).

${ }^{27}$ S. Torquato, B. Lu, and J. Rubinstein, Phys. Rev. A 41, 2059 (1990).

${ }^{28}$ S. Torquato, Phys. Rev. E 51, 3170 (1995).

${ }^{29}$ R. W. Zwanzig, J. Chem. Phys. 22, 1420 (1954).

${ }^{30}$ J. R. Errington, P. G. Debenedetti, and S. Torquato, J. Chem. Phys. 118, 2256 (2003)

${ }^{31}$ L. D. Landau and E. M. Lifshitz, Theory of Elasticity (ButterworthHeinemann, Oxford, 1999).

${ }^{32}$ W. B. Russel, D. A. Saville, and W. R. Schowalter, Colloidal Dispersions (Cambridge University Press, Cambridge, 1989).

${ }^{33}$ D. L. Feke, N. D. Prabhu, J. A. Mann, and J. A. Mann, J. Phys. Chem. 88, 5735 (1984).

${ }^{34}$ R. Hogg, T. W. Healy, and D. W. Fuerstenau, Trans. Faraday Soc. 62, 1638 (1966)

${ }^{35}$ J. Israelachvili, Intermolecular and Surface Forces (Academic, San Diego, 1992).

${ }^{36}$ K. L. Johnson, K. Kendall, and A. D. Roberts, Proc. R. Soc. London, Ser. A 324, 301 (1971).

${ }^{37}$ C. S. Hodges, J. A. S. Cleaver, M. Ghadiri, R. Jones, and H. M. Pollock, Langmuir 18, 5741 (2002).

${ }^{38}$ N. W. Ashcroft and N. D. Mermin, Solid State Physics (Holt, Rinehart \& Winston, New York, 1976).

${ }^{39}$ S. Chandrasekhar, Rev. Mod. Phys. 15, 1 (1943).

${ }^{40}$ N. Shahidzadeh-Bonn, P. Vie, X. Chateau, J. N. Roux, and D. Bonn, Phys. Rev. Lett. 95, 175501 (2005).

${ }^{41}$ S. Torquato, Random Heterogeneous Materials (Springer, New York, 2002).

${ }^{42}$ S. Nemat-Nasser and M. Hori, Micromechanics: Overall Properties of Heterogeneous Materials (Elsevier, Amsterdam, 1999).

${ }^{43}$ www.mse.cornell.edu/courses/engri111/modulus.htm

${ }^{44}$ S. Tang, Y. Ma, and C. Shiu, Colloids Surf., A 180, 7 (2001).

${ }^{45}$ S. Tang, J. M. Preece, C. M. McFarlane, and Z. Zhang, J. Colloid Interface Sci. 221, 114 (2000).

${ }^{46}$ A. D. Dinsmore, E. R. Weeks, V. Prasad, A. C. Levitt, and D. A. Weitz, Appl. Opt. 40, 4152 (2001).

${ }^{47}$ M. Lattuada, H. Wu, and M. Morbidelli, J. Colloid Interface Sci. 268, 106 (2003).

${ }^{48}$ M. Rottereau, J. C. Gimel, T. Nicolai, and D. Durand, Eur. Phys. J. E 15, 133 (2004).

${ }^{49}$ J. Durant, Sands, Powders and Grains (Springer, Berlin, 2000).

${ }^{50}$ T. W. Lambe and R. V. Whitman, Soil Mechanics (Wiley, New York, 1969).

${ }^{51}$ B. K. Chakrabarti and L. G. Benguigui, Statistical Physics of Fracture and Breakdown in Disordered Systems (Clarendon, Oxford, 1997).

${ }^{52}$ A. Wouterse and A. P. Philipse, J. Chem. Phys. 125, 194709 (2006). 